\title{
Deep sclerectomy with collagen implant: medium term results
}

\author{
Marc E Karlen, Enrique Sanchez, Corinne C Schnyder, Michel Sickenberg, \\ André Mermoud
}

\begin{abstract}
Aims-To study prospectively the success rate and complications of deep sclerectomy with collagen implant (DSCI), a new non-penetrating filtration procedure.

Methods-Non-randomised prospective trial involving consecutive patients. 100 eyes of 100 patients with medically uncontrolled primary and secondary open angle glaucoma. A superficial scleral flap was raised and a deep sclerectomy was performed in the scleral bed. Schlemm's canal was opened and dissection of the cornea was performed up to Descemet's membrane, at which point aqueous percolated through the remaining trabeculoDescemet's membrane. A collagen implant was sutured radially in the scleral bed. Visual acuity, intraocular pressure (IOP) measurements, and slit lamp examinations were performed before surgery and after surgery at 1 and 7 days, and $1,3,6,9,12,18,24,30$, and 36 months.

Results-The mean follow up period was 17.8 (SD 8.7) months. The mean preoperative IOP was $27.8(8.6) \mathrm{mm} \mathrm{Hg}$; the mean postoperative IOP was $5.7(4.0)$ at day $1,11.2(4.6)$ at month $1,14.0(3.5)$ at month 12, and 13.0 (3.8) at month 36. Complete success rate, defined as an IOP lower than $21 \mathrm{~mm} \mathrm{Hg}$ without medication, was $44.6 \%$ at 36 months. Qualified success rate, defined as an IOP lower than $21 \mathrm{~mm}$ $\mathrm{Hg}$ with medication, was $97.7 \%$ at 36 months. Early postoperative complications included hyphaema in seven patients, wound leak in 10 patients, and subtle choroidal detachment in 11 patients. Goniopuncture was performed in 41 of the patients, and 5-fluorouracil injections were made in 23 patients; cataract progression occurred in seven patients. When comparing the different types of open angle glaucoma, no difference was found in terms of reduction in IOP, number of patients requiring antiglaucoma medications, or success rate.

Conclusions-Deep sclerectomy with collagen implant appears to provide reasonable control of IOP at medium term follow up, with few immediate postoperative complications.

(Br f Ophthalmol 1999;83:6-11)
\end{abstract}

Deep sclerectomy with collagen implant (DSCI) is a non-perforating filtration procedure used for the surgical treatment of medically uncontrolled open angle glaucoma. This procedure was developed in an attempt to avoid the immediate postoperative complications of trabeculectomy. ${ }^{1-7}$ In order to enhance the passage of aqueous from the anterior chamber to the subconjunctival space and to lower the risk of scleral fibrosis, a collagen implant was used at the end of the deep sclerectomy dissection. ${ }^{89}$

In the study described here, we analysed the complications and outcomes of the first 100 eyes that underwent DSCI and that were followed for up to 36 months.

\section{Materials and methods}

One hundred eyes of 100 patients with medically uncontrolled primary and secondary open angle glaucoma underwent DSCI. All study patients were enrolled consecutively. There were 58 male and 42 female patients, all white; mean age was 71 (SD 12.6) years (Table 1). The study was approved by the ethics committee of the University of Lausanne and informed consent was obtained from all participants. Inclusion criteria for the study were primary or secondary open angle glaucoma with uncontrolled intraocular pressure (IOP) while receiving maximal medical therapy, and willingness to be followed at the Jules Gonin Hospital. Uncontrolled glaucoma was defined as an uncontrolled IOP measured with a Goldmann applanation tonometer under maximal tolerable antiglaucomatous therapy with evidence of progression of the optic neuropathy and/or visual field defect. Exclusion criteria were angle closure glaucoma, unwillingness to participate in the study, known allergy to porcine derived products or collagen, previous eye surgery, previous argon laser trabeculoplasty less than 30 days before enrolment in the study, age less than 35 years, and presence of DSCI in the fellow eye.

Before surgery, all patients underwent best corrected visual acuity assessment (Snellen chart at 5 metres), biomicroscopy, gonioscopy, applanation tonometry with the Goldmann tonometer, visual field testing using the G1 program of the Octopus 101, and fundus examination. After surgery the same examinations, except for visual fields, were performed 


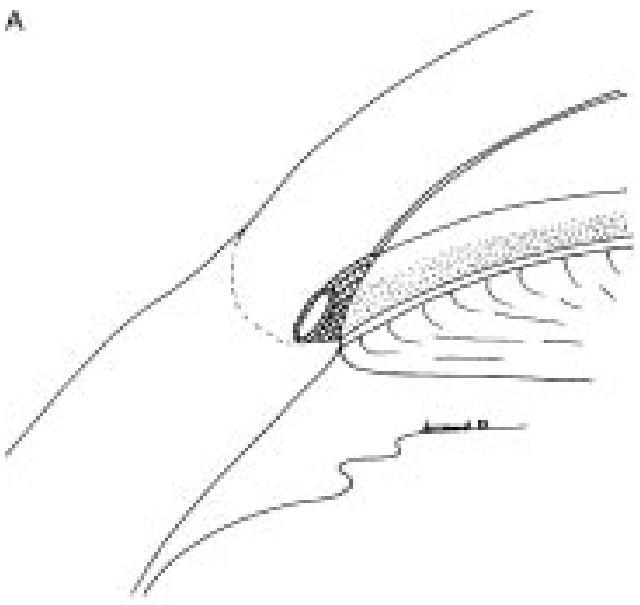

B

Figure 1 (A) Schematic representation if the irido-corneal angle before deep sclerectomy with collagen implant. (B) Schematic representation of the irido-corneal angle after deep sclerectomy with collagen implant. The aqueous humour flows out of the anterior chamber through the trabeculo-Descemet's membrane, fills the scleral space (the pathway of which is maintained with the collagen implant), and then flows to the subconjunctival space. tion was considered to be present when flare could be seen by biomicroscopy; choroidal detachment was considered present when seen in the peripheral retina, using an indirect ophthalmoscope; in the postoperative follow up, cataract was either observed as a direct consequence of filtration surgery and termed "surgery related cataract", or appeared progressively and was called "cataract progression". Surgery related cataract was defined by a rapid decrease (1 month) of visual acuity and mainly the development of cortical opacity, whereas cataract progression was defined as slow progressive decrease in visual acuity of more than two lines (Snellen chart) due to lens opacification, mainly nuclear sclerosis.

SURGICAL PROCEDURES

All surgeries were performed by one experienced surgeon (AM), using retrobulbar anaesthesia consisting of $2-4 \mathrm{ml}$ of a solution of bupivacaine $0.75 \%$, xylocaine $4 \%$, and hyaluronidase $50 \mathrm{U}$.

DEEP SCLERECTOMY WITH COLLAGEN IMPLANT

(FIG 1)

A superior rectus muscle suture was placed. The conjunctiva and Tenon's capsule were then opened in the upper fornix and the sclera exposed; careful haemostasis using wet field cautery was performed. A one third scleral thickness limbus based scleral flap measuring $5 \times 5 \mathrm{~mm}$ was dissected. This superficial scleral flap was dissected $1 \mathrm{~mm}$ into clear cornea. A triangle of deep sclera was then removed, leaving a thin layer of deep sclera over the choroid posteriorly. Anteriorly, the dissection was done down to the Schlemm's canal, which was unroofed; more anteriorly, the excision of corneal stroma was done down to Descemet's membrane. At this stage of the procedure, aqueous humour was seen to percolate through the remaining thin trabeculo-Descemet's membrane. The cylindrical collagen implant drainage device measuring $1 \times 1 \times 2.5 \mathrm{~mm}$ was then placed radially in the centre of the deep sclerectomy dissection and secured with a single 10-0 nylon suture to the thin remnant, posterior to the Schlemm's canal, scleral layer (Fig 2). The superficial scleral flap was repositioned over the collagen implant drainage device and secured with a single 10-0 nylon suture, and the knots buried. No tension was applied to these two sutures because there is no need for scleral flap resistance since the aqueous outflow resistance is located at the trabeculo-Descemet's membrane. The conjunctiva and Tenon capsule were carefully closed with a running 9-0 Vicryl suture.

Postoperatively, patients were treated with topical dexamethasone $0.1 \%$, neomycin $0.35 \%$, and polymyxin $6000 \mathrm{U} / \mathrm{ml}$ (Maxitrol) three times a day for 2 weeks, and then with topical fluorometholone $1 \mathrm{mg}$ three times a day for 3-6 months.

When a perforation of the thin trabeculoDescemet's membrane occurred during the corneal stroma dissection, the surgery was converted into a standard trabeculectomy, with a rectangular resection of the trabeculum, followed by a basal iridectomy.
POAG = primary open angle glaucoma $;$ PEXG = pseudoexfoliation glaucoma; PSPHG = open angle glaucoma in pseudophakic patients; $\mathrm{NPG}=$ normal pressure glaucoma $\mathrm{PIGG}=$ pigmentary glaucoma; OAGA: open angle glaucoma in aphakic patients. 


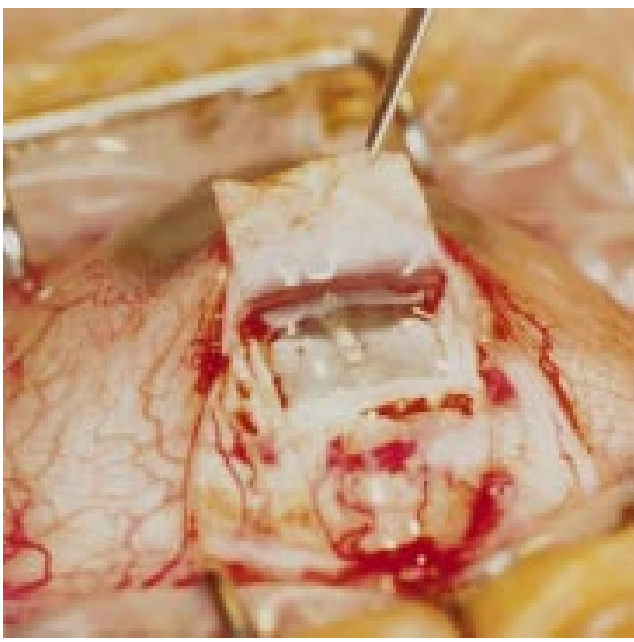

Figure 2 Photograph of collagen implant being placed after deep sclerectomy dissection. Schlemm's canal has been opened, anterior trabeculum and Descemet's membrane are exposed, and aqueous is filtering through the remaining membrane.

Surgery was considered a complete success when IOP was $\leqslant 21 \mathrm{~mm} \mathrm{Hg}$ without glaucoma medication, a qualified success when IOP was $\leqslant 21 \mathrm{~mm} \mathrm{Hg}$ with glaucoma medication, and a failure when IOP was $>21 \mathrm{~mm} \mathrm{Hg}$ with or without glaucoma medication, or when an eye required further glaucoma drainage surgery, developed phthisis bulbi, or lost light perception. Three measurements of the IOP were performed to determine the mean IOP; when this mean IOP was $>21 \mathrm{~mm} \mathrm{Hg}$, it was considered as failure.

When the filtering bleb at any postoperative visit was encysted or showed signs of fibrosis, subconjunctival injections of $5 \mathrm{mg}$ of 5-fluorouracil (5-FU) were performed in the lower quadrant, opposite the deep sclerectomy with collagen implant. The subconjunctival injections consisted on $0.1 \mathrm{ml}$ of a $50 \mathrm{mg} / \mathrm{ml}$ solution of $5-\mathrm{FU}(250 \mathrm{mg} / 5 \mathrm{ml}$ fluorouracil, Roche (Basle, Switzerland). Subconjunctival 5-FU injections were repeated up to seven times if needed.

Goniopuncture with the Nd:YAG laser (Microruptor II, Lasag AG, Thun, Switzerland) was performed when the filtration through the trabeculo-Descemet's membrane was suspected to be insufficient, either because

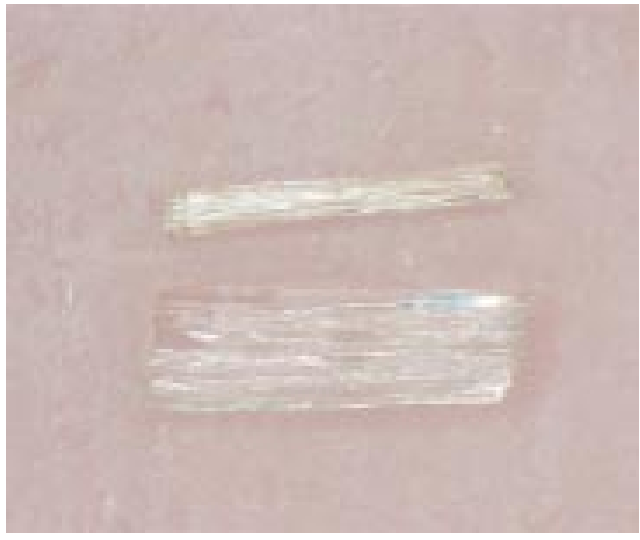

Figure 3 Dry lyophilised collagen implant and collagen implant after hydration.

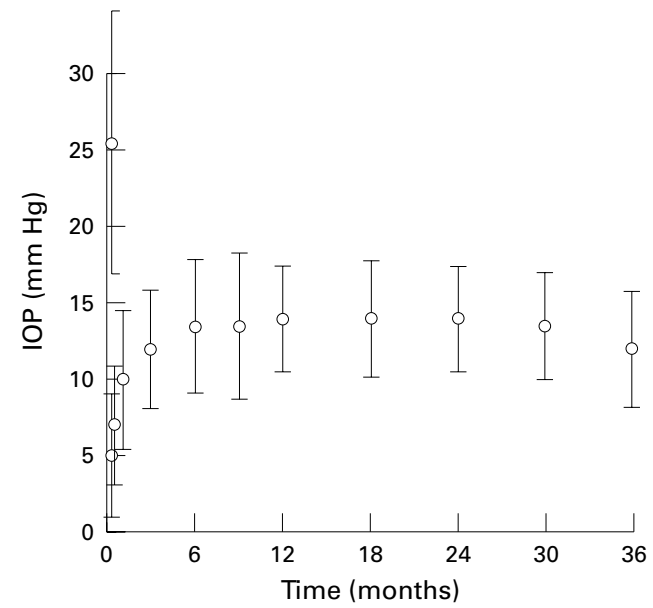

Figure 4 Intraocular pressure before and after deep sclerectomy with collagen implant.

of a shallow filtration bleb or elevated IOP. The laser treatment was performed using a Lasag-15 gonioscopy contact lens (CGA1). Goniopuncture was performed using the free running $Q$ switch mode with energy ranging from 2 to $4 \mathrm{~mJ}$. The goal of the laser treatment was to create a small hole in the trabeculoDescemet's membrane, which is technically similar to posterior capsulotomy after cataract surgery. Goniopuncture allows direct passage of aqueous from the anterior chamber to the filtration bleb and transforms the nonperforating filtering surgery into a perforating filtering surgery. After laser treatment, patients were treated with topical prednisolone acetate (Predforte) three times a day for 3 days. Laser goniopuncture was considered a success when final IOP was $<18 \mathrm{~mm} \mathrm{Hg}$, or when the decrease in IOP was $>5 \mathrm{~mm} \mathrm{Hg}$ with initial pressure $\leqslant 23 \mathrm{~mm} \mathrm{Hg}$.

GLAUCOMA COLLAGEN IMPLANT

The cylindrical collagen implant is manufactured by Staar Surgical AG, Nidau, Switzerland. It measures $2.5 \mathrm{~mm}$ in length and $1 \mathrm{~mm}$ in diameter. The collagen implant is processed from lyophilised American porcine scleral collagen, which is sterilised by a radiation procedure. Microbiological analyses performed

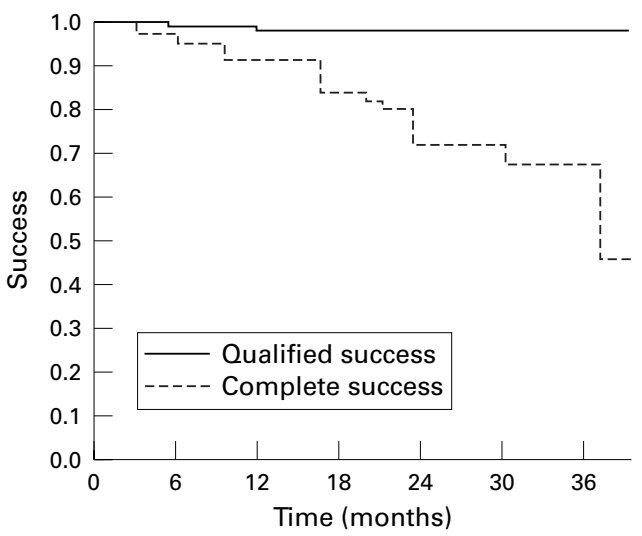

Figure 5 Long term cumulative complete success probability and qualified success probability of deep sclerectomy with collagen implant, using Kaplan-Meier life table analysis. 


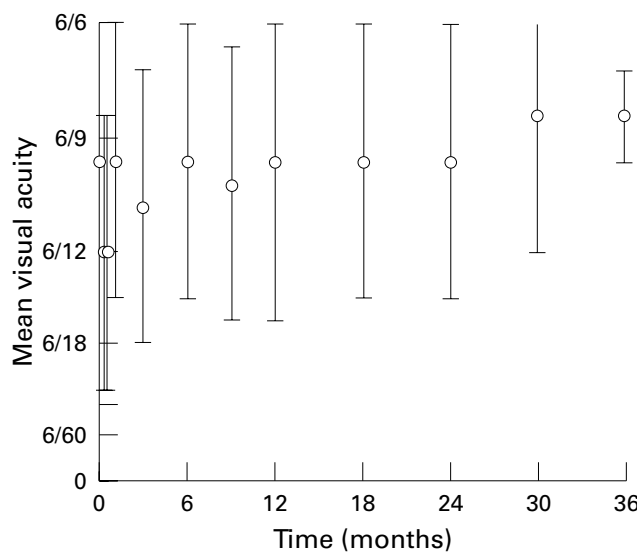

Figure 6 Evolution of the mean visual acuity after DSCI.

by Professor Nordmann, Hôpital Antoine Beclere in Paris on $30 \mathrm{~g}$ of raw collagen material have not revealed the presence of bacteria, virus, mycological organisms, and parasites. The water content of the hydrated device is 99\% (Fig 3). The collagen device is resorbed within 6-9 months after surgery as was documented in a previous ultrasonographic study. ${ }^{10} 11$

STATISTICAL ANALYSIS

Results were analysed using the Student's $t$ test for comparison of means, $\chi^{2}$ analysis for $2 \times 2$ tables, and Kaplan-Meier survival curves for long term success rate analysis; for comparison between groups, the Wilcoxon test was used.

\section{Results}

Of 106 patients on whom DSCI was planned, six were found to have a perforation of the thin trabeculo-Descemet's membrane at the time of surgery, so the procedure had to be converted into a trabeculectomy. These six patients were excluded from the study. In the first 10 deep sclerectomies, iatrogenic perforation of the trabeculo-Descemet's membrane during sur-

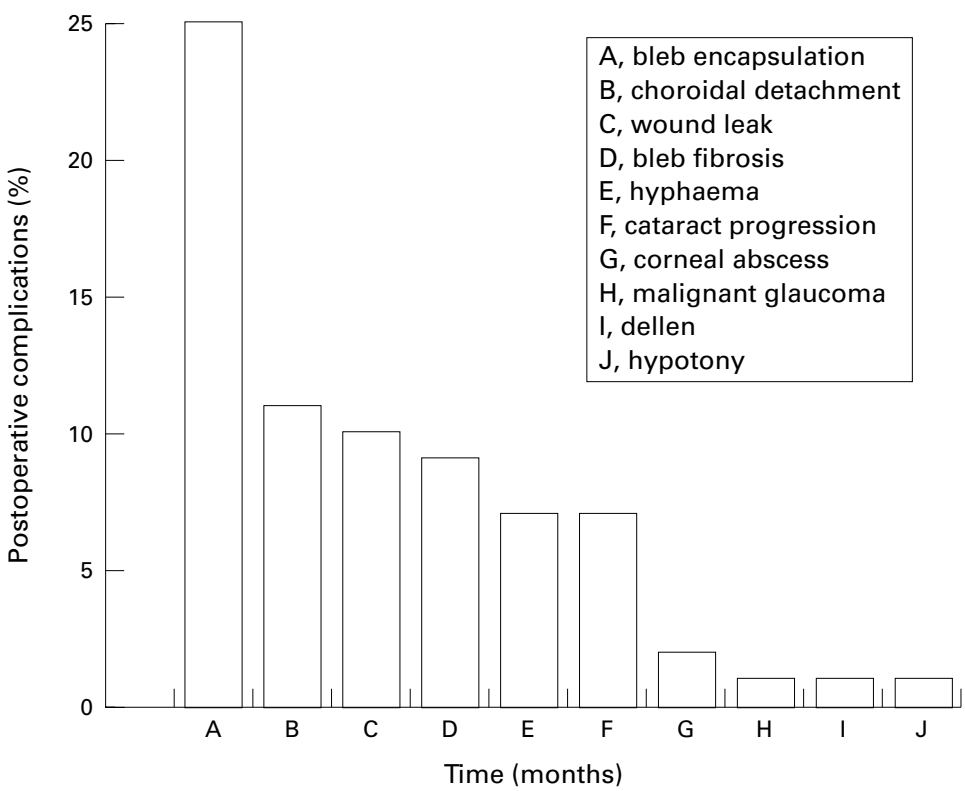

Figure 7 Postoperative complications. gery occurred three times $(30 \%)$, whereas in the subsequent 96 surgeries the membrane was perforated only three times $(3.1 \%)$.

The mean follow up time was 17.8 (SD 8.7) months. The IOP at 3 months was reduced by $55.4 \%$ (12.4 (3.9) versus $27.8(8.6) \mathrm{mm} \mathrm{Hg}$ ), and at 36 months was reduced by $53.2 \%(13.0$ (3.8) versus $27.8(8.6) \mathrm{mm} \mathrm{Hg}$ ), thus showing stability of the IOP postoperatively (Fig 4).

Eyes treated with DSCI had a complete success rate at 36 months of $44.6 \%$; the qualified success rate was $97.7 \%$ at 36 months (Fig 5). The mean number of medications per patient required to control IOP was reduced from 2.2 (0.7) before DSCI to $0.2(0.4)$ after DSCI, ( $p$ $=0.001)$. On the first postoperative day, best corrected visual acuity (UK notation) was $6 / 12$ $(6 / 18)$. Visual acuity returned to preoperative levels 1 week after surgery and remained stable for the following 3 years (Fig 6). Postoperative complications that occurred are listed (Fig 7). We observed no shallow or flat anterior chamber, no anterior chamber inflammation, no bleb related endophthalmitis, and no surgery induced cataract. However, $7 \%$ of patients showed a progression of pre-existing cataract. Bleb fibrosis and encapsulation were treated with 5-FU subconjunctival injections. Subconjunctival injections of 5-FU were made in 23 patients who underwent DSCI. The mean number of injections per patient was 2.9 (2.2) and the mean time between operation and 5-FU injection was 1.9 (2.7) months.

Goniopuncture with the Nd:YAG laser was performed in 41 patients at various times after DSCI. The mean time between surgery and goniopuncture was 11.0 (7.5) months. Mean IOP before goniopuncture was $22.2(7.0) \mathrm{mm}$ $\mathrm{Hg}$ and decreased to 12.3 (5.8) $\mathrm{mm} \mathrm{Hg}$, the success rate being $83 \%$.

Comparing the result of the three main glaucoma categories (primary open angle glaucoma (52 patients), pseudoexfoliative glaucoma (20 patients), and open angle glaucoma in pseudophakic patients (14 patients), it was found that patients with primary open angle glaucoma were younger, 68.9 (12.1) versus 75.7 (7.8) and 76.4 (10.5), for pseudoexfoliative glaucoma and open angle glaucoma in pseudophakia, respectively. There was no significant difference in terms of postoperative IOP, qualified success rate, or number of postoperative glaucoma medications. However, there is a tendency of lower medium term success rate in the PSPHG group (Table 2).

\section{Discussion}

In an attempt to avoid the numerous postoperative complications of trabeculectomy, such as hypotony, flat anterior chamber, and choroidal detachment, some authors proposed a tight closure of the scleral flap with secondary suture lysis with the argon laser. ${ }^{12}$ More recently, for the same reason, several techniques of nonperforating filtration surgery have been described. Zimmerman et $a l^{13}{ }^{14}$ reported good results of non-penetrating trabeculectomy in phakic and aphakic patients. Arenas ${ }^{15}$ developed a technique called ab externo trabeculectomy, and reported a success rate of $88 \%$. 
Table 2 Comparison of results of deep sclerectomy with collagen implant between primary open angle glaucoma (POAG), pseudoexfoliative glaucoma (PEXG), and open angle glaucoma in pseudophakic patients (PSPHG)

\begin{tabular}{|c|c|c|c|c|}
\hline & $P O A G$ & $P E X G$ & PSPHG & $p$ Value \\
\hline Age (years) (SD) & $68.9(12.1)$ & $75.7(7.8)$ & $76.4(10.5)$ & $<0.03^{\star}$ \\
\hline Preoperative IOP (mm Hg) (SD) & $26.3(7.7)$ & $30.5(8.1)$ & $28.9(10.0)$ & 0.058 \\
\hline \multicolumn{5}{|l|}{ Postoperative IOP (mm Hg) (SD) } \\
\hline 12 months & $14.3(3.2)$ & $13.2(3.5)$ & $12.5(3.4)$ & NS \\
\hline 24 months & $12.9(3.3)$ & $13.3(3.5)$ & $13.4(4.9)$ & NS \\
\hline Complete success rate (\%) 30 months & 75.8 & 71 & 57 & NS \\
\hline Qualified success rate (\%) 30 months & 98 & 100 & 100 & NS \\
\hline Number of preoperative glaucoma medications (SE) & $2.1(0.6)$ & $2.4(0.8)$ & $2.4(0.7)$ & NS \\
\hline Number of postoperative glaucoma medications (SE) & $0.2(0.3)$ & $0.2(0.4)$ & $0.3(0.4)$ & NS \\
\hline Number of patients having 5-FU injections & $11 / 52(21 \%)$ & $4 / 20(20 \%)$ & $3 / 14(21 \%)$ & NS \\
\hline Number of patients having goniopuncture & $18 / 52(21 \%)$ & $10 / 20(50 \%)$ & $6 / 64(43 \%)$ & NS \\
\hline
\end{tabular}

* POAG patients are significantly younger than those with PEXG or PSPHG.

$\mathrm{NS}=$ not significant; IOP = intraocular pressure; SE = standard error; SD = standard deviation; 5-FU = 5-fluorouracil.

Other authors who used the ab externo technique reported control of IOP in $85.8 \%$ to $90 \%$ of patients. ${ }^{16-18}$ Stegmann $^{19}$ described a similar technique, in which the scleral space was filled with a viscoelastic substance, and reported complete success in $61 \%$ of patients and qualified success in $77 \%$ at 26 months of follow up.

To enhance the filtration of deep sclerectomy, Kozlov et $a t^{8}$ and Fyodorov ${ }^{20}$ described the use of a collagen implant placed within the scleral bed. Similarly, in a prospective and randomised study, we achieved better surgical outcome when the collagen implant was used. ${ }^{9}$

The major advantage of DSCI is that it precludes the sudden hypotony which occurs following trabeculectomy by creating progressive filtration of aqueous humour from the anterior chamber to the subconjunctival space, without perforation of the eye..$^{10}$ In an experimental model, Mermoud and Vaudaux ${ }^{21}$ studied the aqueous dynamics through the remaining thin trabeculo-Descemet's membrane, and found that the outflow resistance was low but sufficient to avoid the immediate postoperative complications often seen after trabeculectomy.

The short term success of DSCI has been reported to be good. Demailly et al reported a mean decrease in IOP of 9.1 (7.1) $\mathrm{mm} \mathrm{Hg}$ after 219 DSCI procedures. ${ }^{22}$ Using Kaplan-Meier survival analysis, they reported a success rate without glaucoma medication of $89 \%$ at 6 months, and $75.6 \%$ at 16 months; with glaucoma medication, their success rate increased to $97 \%$ at 6 months, and $79 \%$ at 16 months. Kozlov et $a l^{8}$ reported an $85 \%$ success rate, but no information regarding success criteria or follow up is available. The mid-term (36 months) success of DSCI remains satisfactory. The complete success rate of DSCI was $97 \%$ at 6 months, $71.4 \%$ at 24 months, and $44.6 \%$ at 36 months. With glaucoma medication, the success rate was $99 \%$ at 6 months, and $97.7 \%$ at 36 months. Interestingly, in our study, although the preoperative age and IOP was different in the three main glaucoma groups (Table 2), the postoperative success rates were similar. This may be explained by the reproducibility of the trabeculoDescemet's membrane resistance which allows similar postoperative IOP regardless of preoperative levels. For other types of glaucoma such as pigmentary glaucoma, aphakic glaucoma, traumatic glaucoma, and other more rare secondary open angle glaucomas, no difference could be found in terms of postoperative results, but the number of cases was too small for statistical analysis.

Except for the first week after surgery, visual acuity was generally unaffected by DSCI. This phenomenon may be explained by the nonperforation of the eye, which avoids inflammation, use of mydriatics, and sudden hypotony. Furthermore, visual acuity remains good because no surgery related cataracts developed after DSCI. The $7 \%$ of cataract progression observed in this study corresponds to that observed in a matched age population (unpublished data).

The major intraoperative complication of this surgery was perforation of the thin trabeculo-Descemet's membrane during the deep sclerectomy dissection. This was common in the learning phase of deep sclerectomy, occurring in three of the first 10 surgeries $(30 \%)$; but with increased surgical experience this complication became rare: only three surgeries $(3.1 \%)$ of the subsequent 96 deep sclerectomies resulted in perforation.

The results of the patients who had perforation of the trabeculo-Descemet's membrane were not analysed in this paper because they were reported in another study. ${ }^{23}$ The postoperative complications and final outcome of patients with intraoperative perforation of the trabeculo-Descemet's membrane are similar to those of patients who undergo standard trabeculectomy. ${ }^{23}$

The early postoperative complications included the presence of subtle hyphaema in $7 \%$ of our cases. The very small amount of blood in the anterior chamber was explained by a probable blood reflux from the scleral bed through the anterior trabeculum.

Two patients presented with a voluminous filtration bleb with secondary infiltrated dellen. Both patients were considered to have corneal abscess and were treated with topical antibiotics.

Malignant glaucoma is a rare complication of ocular surgery. It usually happens after perforation of the globe, but cases of malignant glaucoma after closed eye manipulation such a laser surgery have been described. The case we report here presented with a malignant glaucoma after trabeculectomy in the left eye. DSCI was performed in the right eye 1 year later. In the postoperative course, the patient was treated prophylactically with scopolamine (hyoscine). One month later, the anterior 
chamber remained deep and the IOP controlled. The scopolamine was then discontinued and the patient developed malignant glaucoma with shallow anterior chamber, rotation of the ciliary body assessed by ultrasound biomicroscopy, and elevated IOP. The patient was successfully treated with scopolamine for another 2 months.

Although DSCI is a safe procedure with few immediate postoperative complications, careful long term follow up is necessary. In our series, 34 patients developed fibrosis or an encysted bleb. These patients were treated with subconjunctival injections of 5-FU. The mean number of injections required per patient (2.9 (SD 2.1)) was much lower than in previous studies using 5-FU after trabeculectomies (7-21 injections). ${ }^{24}$ The majority of $5-\mathrm{FU}$ injections were made during the first 2 postoperative months.

Goniopunctures with the Nd:YAG laser were performed in $41 \%$ of the patients. Goniopuncture was performed shortly after DSCI because there was insufficient percolation of aqueous humour at the trabeculo-Descemet's membrane, probably due to the lack of surgical dissection. When goniopunctures were required at a later time (more than 9 months after initial surgery), low filtration was probably the result of fibrosis of the trabeculo-Descemet's membrane, since goniopuncture resulted in increased filtration of aqueous humour and decreased IOP. The success rate of Nd:YAG laser goniopuncture was satisfactory, with an immediate reduction in mean IOP of $43.7 \%$ (from 22.2 (7.0) to 12.5 (5.8) $\mathrm{mm} \mathrm{Hg}$ ). By opening the trabeculo-Descemet's membrane, however, goniopuncture transformed a nonperforating filtration procedure into a perforating one. Because this perforation can lead to the classic complications (flat anterior chamber and choroidal detachment) of trabeculectomy when performed early after DSCI, we usually wait 3 weeks or more before performing goniopuncture. Although the potential risk of late bleb related endophthalmitis may be increased after goniopuncture, no such case occurred in our study.

Kozlov et $a l^{8}$ also reported early and late postoperative ocular hypertension, and also performed goniopunctures with the Nd:YAG laser, and they achieved a success rate ranging from 85 to $94 \%$. Demailly et $a l_{,}^{22}$ in their series of 219 DSCI procedures, performed 90 goniopunctures with the Nd:YAG laser, of which 61 $(68 \%)$ were successful. These results compare favourably with our success rate of $85 \%$.

Several questions about DSCI remain unanswered: What is the mechanism of aqueous percolation through the thin trabeculoDescemet's membrane? What tissue replaces the collagen drainage device? Is there subchoroidal resorption of aqueous through the thin scleral layer that is left in the deep scleral bed? Does aqueous drain into Schlemm's canal and the aqueous vein? To answer these questions, further studies are needed. Furthermore, long term (5-10 years) clinical follow up studies are necessary.
In conclusion, DSCI appears to be a promising new non-perforating filtration procedure. In our hands, the success rate after 3 years of follow up was satisfactory for all types of primary and secondary open angle glaucoma studied. The immediate postoperative complication rate was low, and visual acuity was almost unaffected. Preservation of the thin trabeculo-Descemet's membrane, however, is technically challenging, particularly before the surgeon gains experience with this procedure. In addition, careful postoperative examinations are needed to identify and treat early and late postoperative ocular hypertension with the Nd:YAG laser, as well as to identify and treat bleb fibrosis and encysted blebs with subconjunctival injections of 5-FU.

Presented in part at the annual meeting of the American Academy of Ophthalmology, San Francisco, California, October 1997.

Dr Mermoud is a consultant for Staar Surgical AG, Nidau, Switzerland.

1 Watson PG, Jakeman C, Ozturk M, et al. The complications of trabeculectomy (a 20-year follow-up) Eye 1990;4:42538.

2 Kao SF, Lichter PR, Musch DC. Anterior chamber depth following filtration surgery. Ophthalmic Surg 1989;20:332-6.

3 Stewart WC, Shields MB. Management of anterior chamber depth after trabeculectomy. Am f Ophthalmol 1988;106:414 .

4 Brubaker RF, Pederson JE. Ciliochoroidal detachment. Surv Ophthalmol 1983;27:281-9.

5 Gressel MG, Parrish RK II, Heuer DK. Delayed nonexpulGressel MG, Parrish RK II, Heuer DK. Delayed nonexpul-
sive suprachoroidal hemorrhage. Arch Ophthalmol 1984; 102:1757-60

6 Ruderman JM, Harbin TS Jr, Campbell DG. Postoperative suprachoroidal hemorrhage following filtration procedures. Arch Ophthalmol 1986;104:201-5.

7 Freedman J, Gupta M, Bunke A. Endophthalmitis after trabeculectomy. Arch Ophthalmol 1978;96:1017-18.

8 Kozlov VI, Bagrov SN, Anisimova SY, et al. Nonpenetrating deep sclerectomy with collagen. Eye Microsurgery (in Russian) 1990;3:44-6.

9 Sanchez E, Schnyder CC, Sickenberg M, et al. Deep sclerectomy: results with and without collagen implant. Int Ophthalmol 1997;20:157-62.

10 Chiou AGY, Mermoud A, Hédiguer SEA, et al. Ultrasound biomicroscopy of eyes undergoing deep sclerectomy with collagen implant. Br f Ophthalmol 1996;80:541-4.

11 Chiou AGY, Mermoud A, Underdahl JP, et al. An ultrasound biomicroscopic study of eyes after deep sclerectomy with biomicroscopic study of eyes after deep sclerectom
collagen implant. Ophthalmology 1998;105:746-50.

12 Melamed S, Ashkenazi I, Glovinski J, et al. Tight scleral flap trabeculectomy with postoperative laser suture lysis. Am f Ophthalmol 1990;109:303-9.

13 Zimmerman TJ, Kooner KS, Ford VJ, et al. Effectiveness of nonpenetrating trabeculectomy in aphakic patients with glaucoma. Ophthalmic Surg 1984;15:44-50.

14 Zimmerman TJ, Kooner KS, Ford VJ, et al. Trabeculectomy vs non-penetrating trabeculectomy: a retrospective study of two procedures in phakic patients with glaucoma. Ophthalmic Surg 1984;15:734-40.

15 Arenas E. Trabeculectomy ab-externo. Highlights of Ophthalmology 1991;19:59-66.

16 Gierek A, Szymanski A. Results of deep sclerectomy for open-angle glaucoma. Folia Ophthalmol (Leipzig) 1987;12: 227-9.

17 Tavano G, Chabin T, Barrut JM. Hémitrabéculectomie ab externo non invasive. Bull Soc Ophtalmol Fr 1993;93:749-50.

18 Tanihara H, Negi A, Akimoto $M$, et al. Surgical effects of trabeculotomy ab externo on adult eyes with primary open ngle glaucoma and pseudoexfoliation syndrome. Arch Ophthalmol 1993;111:1653-61.

19 Stegmann RC. Visco-canalostomy: a new surgical technique for open angle glaucoma. An Inst Barraquer, Spain 1995;25: 229-32

20 Fyodorov SN. Non penetrating deep sclerectomy in openangle glaucoma. Eye Microsurgery (in Russian) 1989:52-5.

21 Mermoud A, Vaudaux J. Aqueous humor dynamics in non penetrating filtering surgery. Invest Ophthalmol Vis Sci 1997;38 (suppl):S1064.

22 Demailly P, Jeanteur-Lunel MN, Berkani M, et al. Non penetrating deep sclerectomy associated with collagen device in primary open angle glaucoma. Middle-term retrospective study. F Fr Ophtalmol 1996;11:659-66.

23 Sanchez E, Schnyder CC, Mermoud M. Résultats comparatifs de la sclérectomie profonde transformée en trabéculectomie et de la trabéculectomie classique. Klin Monatsbl Augenheilkd 1997;210:261-4.

24 Fluorouracil Filtering Surgery Study Group. Fluorouracil Filtering Surgery Study one-year follow-up. Am $\mathcal{F}$ Ophthalmol 1989;108:625-35. 